\title{
Efficacy of robotic versus laparoscopic approach in spleen preserving distal pancreatectomy for benign or low-grade malignant pancreatic tumors: study protocol for a randomized controlled trial
}

\section{Feng TIAN}

Chinese Academy of Medical Sciences and Peking Union Medical College Institute of Basic Medical

Sciences https://orcid.org/0000-0002-0244-4719

Jun Lu

Peking Union Medical College Hospital

Cheng-xi Liu

Peking Union Medical College Hospital

Jun-chao Guo ( $\nabla$ pumch_research@163.com )

Peking Union Medical College Hospital

\section{Study protocol}

Keywords: Benign pancreatic tumor, Low grade malignant, Robotic distal pancreatectomy, Laparoscopic distal pancreatectomy, Spleen preservation

Posted Date: September 28th, 2020

DOI: https://doi.org/10.21203/rs.3.rs-35070/v1

License: (c) (1) This work is licensed under a Creative Commons Attribution 4.0 International License.

Read Full License 


\section{Abstract}

\section{Background}

Based on previous meta-analyses and comparative evidence, robotic approach might lead to a higher or at least similar spleen-preservation rates when comparing with laparoscopic approach in spleen preserving distal pancreatectomy (SPDP) for benign or low-grade malignant pancreatic tumors ${ }^{1-3}$. But high-quality evidence lacks and controversy exists. Therefore, we planned to initiate a prospective, patient-blinded, randomized, controlled trial (RCT) to compare robotic assisted spleen preserving distal pancreatectomy (RSPDP) with laparoscopic spleen preserving distal pancreatectomy (LSPDP), with special consideration to the success rate of splenic vessel-preserving (Kimura) approach, as well as perioperative variables, and cost analysis.

Methods

Patients with benign or low-grade malignant pancreatic tumors are potential candidates for this RCT. Enrolled participants are randomized into RSPDP (observational arm) or LSPDP (control arm) groups as a ratio of 1:1. The primary outcome is the success rate of splenic vessel-preserving (Kimura) approach. The secondary outcomes include total success rate of spleen preserving (Kimura and Warshaw approaches), intraoperative variables, time to functional recovery, postoperative complications, mortality, and cost/efficacy analysis.

Discussion

We plan to conduct a randomized controlled trial to detect the potential advantages of robotic approach in spleen vessels preservation compared with traditional laparoscopic approach. RSPDP may improve spleen vessels preservation rate in selected patients.

Trial registration

Chinese Clinical Trial Registry, No: ChiCTR2000029177. Registered on 18 January, 2020.

\section{Administrative Information}

Note: the numbers in curly brackets in this protocol refer to SPIRIT checklist item numbers. The order of the items has been modified to group similar items (see http://www.equator-network.org/reportingguidelines/spirit-2013-statement-defining-standard-protocol-items-for-clinical-trials/). 


\section{Title $\{1\}$ \\ Efficacy of robotic versus laparoscopic approach in spleen preserving distal pancreatectomy for benign or low-grade malignant pancreatic tumors: study protocol for a randomized controlled trial}

Trial registration $\{2 a \quad$ Chinese Clinical Trial Registry, No: ChiCTR2000029177. and $2 \mathrm{~b}\}$.

Registered on 18 January 2020.

Protocol version $\{3\} \quad$ Version 2.0. Date: 28 April, 2020.

Funding $\{4\} \quad$ Department of General Surgery, Peking Union Medical College Hospital \& Chinese Academy of Medical Sciences (PUMCH). To the date, without any other funding yet.

Author details $\{5 \mathrm{a}\} \quad$ All of the protocol contributors are affiliated to Peking Union Medical College Hospital \& Chinese Academy of Medical Sciences.

Pro. Jun-chao Guo is the corresponding author.

Name and contact information for the trial sponsor $\{5 b\}$

Role of sponsor $\{5 \mathrm{c}\}$
Name: Department of General Surgery, Peking Union Medical College Hospital \& Chinese Academy of Medical Sciences. Contact information: Tel: 01069152600;

The study sponsor plays an important role in study monitor, data management, and interpretation of data. Moreover, it has the ultimate authority over any of these activities.

\section{Introduction}

\section{Background and rationale $\{6 a\}$}

Distal pancreatectomy (DP) is traditionally combined with splenectomy. Splenic preservation DP (SPDP) has gained popularity for resection of benign or low-grade malignant lesions of the distal pancreas due to the benefit of avoiding short-term (Overwhelming post-splenectomy infection, OPSI) and long-term postsplenectomy complications (hypercoagulabitlity, increased risk of malignant tumors) ${ }^{4,5}$. Typical indications for SPDP include: mucinous cystic neoplasm (MCN), low-grade pancreatic neuroendocrine tumor (pNET), benign intraductal papillary mucinous neoplasm (IPMN), and solid pseudopapillary neoplasm (SPN). Kimura approach and Warshaw technique are two commonly reported approaches to $\mathrm{SPDP}^{6}$. Though technically challenging, Kimura procedure is the most ideal choice because it preserves the native vasculature of the spleen and could avoid complications such as splenic infarction, abscess or perigastric collateral/ submucosal varices (66\% varices rate reported by Boselli et al.) after Warshaw technique $e^{5,7}$.

Robotic-assisted DPs have been reported increasingly. Retrospective studies have reported that the splenic vessel-preserving DP rate ranged from 50\% 72.3\% and the total SP rate (splenic vessel-preserving or sacrificing) ranged from $72.5 \% \sim 96.8 \%$ via robotic approach. Also, published literature revealed that RSPDP is associated with potentially increased splenic vessel-preserving DP rate or SP rate than that of LSPDP $(12.9 \% \sim 51.5 \%$ or $39.4 \% \sim 84.1 \%$, respectively). Whereas comparable perioperative outcomes have 
been reported between the two approaches, including operative times, conversion to laparotomy, postoperative length of stay, pancreatic fistula, blood transfusion, or readmission rate ${ }^{8-13}$. Nevertheless, randomized controlled trial comparing outcomes between RSPDP and LSPDP are lacking. Given the advantages of the robotic surgical platform in stable magnified three-dimensional view, EndoWrist technique, and tremor elimination, it's necessary to conduct a RCT to verify the superiority of robotic approach.

\section{Objectives $\{7\}$}

This study is designed to test the hypothesis that RSPDP, compared with LSPDP, might increase the spleen preservation rate and splenic vessels preservation rate for benign or low-grade malignant pancreatic tumors. Meanwhile, via the study, we try to promote the wide implementation of robotic surgeries for patients with DPs, especially candidates need SPDPs. Also, the process of the study would help training young generation of pancreatic surgeons.

\section{Trial design $\{8\}$}

This study is a single-institution, patient-blinded, parallel, noninferiority, randomized controlled trial. The two comparing arms include: RSPDP as observational group, in which spleen preserving distal pancreatectomy is performed via robotic approach. LSPDP as controlled group, in which spleen preserving distal pancreatectomy is performed via laparoscopic approach. Participants are allocated with a $1 \otimes 1$ ratio into the two arms.

\section{Methods: Participants, Interventions And Outcomes}

\section{Study setting $\{9\}$}

From 1 June, 2020 to 30 June, 2023, candidate patients with suspected benign or low-grade malignant pancreatic tumors will be scanned for enrollment in a tertiary teaching hospital (Peking Union Medical College Hospital, PUMCH). A total of 134 patients are needed with a $5 \%$ of expulsion rate. Besides enrollment, patients' allocation, follow-up and data analysis are also performed in PUMCH (Fig. 1).

\section{Eligibility criteria $\{10\}$}

Study candidates received enhanced computed tomography (CT) scans plus three-dimensional reconstruction of the abdomen and pelvic to evaluate the pancreatic tumor and its adjacencies. Cardiopulmonary function was also assessed to meet the requirement of upper abdominal surgeries.

Inclusion criteria include:

a. Patients suspected with pancreatic benign or low-grade malignant tumors preoperatively;

b. Aged 18 to 80;

C. Serous cystic neoplasm with significant and related symptoms 
d. Mucinous cystic neoplasm sized $\geq 3 \mathrm{~cm}$ without any imaging features indicating high-grade dysplasia or cancer, or symptomatic cases without malignancy features.

e. Solid pseudopapillary tumor (SPT) sized $\leq 6 \mathrm{~cm}$;

f. Well-differentiated neuroendocrine tumor sized 1 2cm;

g. Intraductal papillary mucinous neoplasm (IPMN) with out evidence of malignancy.

Exclusion criteria include:
a. Aged $₫ 18$ or $₫ 80$;
b. History of upper abdominal surgical history such as splenectomy, gastrectomy, liver resection, duodenal or pancreatic resection (not including laparoscopic cystectomy);
c. Suspected pancreatic cancer;
d. Pancreatic trauma;
e. With pneumoperitoneum contraindications;
f. With severe heart or pulmonary diseases not fit for surgeries;
g. Failure in obtaining informed consent.

Eligibility of participating surgeons

Surgeons' experience would affect the success rate of Kimura procedure. To control operators' bias, each participating surgeon requires qualified criteria: (1) had experience of 100 pancreatectomies in career, (2) 50 pancreatectomies annually, (3) had experience of more than 50 laparoscopic pancreatectomies, (4) had experience of more than 15 robotic pancreatectomies. One surgical team (Guo, and Tian) plans to perform RSPDP and LSPDP procedures. Newly added surgeons have to meet the above requirements for participation.

\section{Who will take informed consent? $\{26 a\}$}

Co-researchers (Feng Tian and Jun Lu) will obtain the informed consent or assent from potential participants with well designed consent form.

\section{Additional consent provisions for collection and use of participant data and biological specimens $\{26 \mathrm{~b}\}$}

Not applicable. Because in the present trial, there is no plan of collecting or using of participant data and biological specimens.

\section{Interventions}

\section{Explanation for the choice of comparators $\{6 \mathrm{~b}\}$}

The two choices of comparators include, RSPDP as observational group: SPDP is performed via robotic approach. LSPDP as controlled group: SPDP is performed via laparoscopic approach. 


\section{Intervention description $\{11$ a $\}$}

\section{Standardized surgical technique for Kimura approach of SPDP}

A supine position with the head and left side elevated is adapted. Main surgical steps include: Division of the gastrocolic ligament is firstly performed. The short gastric and left gastroepiploic vessels are preserved. Generally, mobilization of the distal pancreas and the contained tumor is started by dissection of the inferior margin of the pancreas. The inferior mesenteric vein may be ligated if difficult to separate from the tumor. Subsequently, the access to the post-pancreatic plane can be achieved after incising the retroperitoneum along the inferior pancreas. The inferior profile of the splenic vein is then dissected and presented. In the following, the supera-pancreatic stereoscopic triangle is dissected, which consisted of common hepatic artery, gastroduodenal artery, superior border of the pancreas, and anterior wall of portal vein (PV). Herein, the root of splenic artery is identified. Subsequently, cautious dissection of the neck portion was performed according to the anatomic landmark of right gastroepiploric vein. Commonly, one to two small tributary vessels from dorsal pancreas to SMV-PV needed to be ligated. After the post-neck tunnel is presented, the pancreatic is transected with stapler or ultrasound scalpel, according to the situation. By then, the distal pancreas is stripped from the splenic vessels to dissociate the pancreatic body-tail completely, during which several small vessel branches need to be ligated. When approaching the spleen hilum, vessel suture may be needed in case of bleeding. Also, transient blocking of the splenic vessels could be performed to control bleeding if necessary. If Kimura approach cannot be completed, Warshaw technique or splenectomy will be considered according to intraoperative situation.

\section{Criteria for discontinuing or modifying allocated interventions $\{11 \mathrm{~b}\}$}

Criteria for discontinuing or modifying include: (1) Participant requests to quit the study after allocation. (2) Post-allocation assessment reveals evidence of unexpected contraindication of the allocated intervention, such as pneumoperitoneum. (3) The main outcome is not recorded or incomplete, which will affect further statistical analysis.

\section{Strategies to improve adherence to interventions $\{11 \mathrm{c}\}$}

The outcomes are observed during operative and postoperative recovery period. Therefore, adherence of the participants is not worried. However, we will pay enough attention to patients' informed consents.

\section{Relevant concomitant care permitted or prohibited during the trial $\{11 \mathrm{~d}\}$}

The research team works out a detailed flow chart to guide the care during the trial (Table 1). We routinely use octreotide (postoperative 3 days), prophylactic analgesics, antibiotics (postoperative one day), blood tests, and proton pump inhibitors according to previous experience. The amylase level of the drainages is tested on postoperative 1, 3, 5, 7 days, respectively. Abdominal and pelvic CT scan is applied before discharge to exclude abdominal fluid collection or abscess.

\section{Provisions for post-trial care $\{30\}$}


We plan to set trial-specific clinic for participants to perform convenient follow-up. Also, we supply hot line service for participants in case of post-trial complications.

\section{Outcomes $\{12\}$}

The primary outcome is defined as the success rate of splenic vessel-preserving (Kimura) approach.

Secondary outcomes include total success rate of spleen preserving (Kimura and Warshaw approaches), intraoperative variables (such as operative time, estimated blood loss $₫$ conversion to open, transfusion, remnant closure), time to functional recovery (VAS score, time to first flatus and intake, LOS), postoperative complications (pancreatic fistula, hemorrhage, infection, chylous fistula, fluid collection, DGE, wound infection, lung infection, or thrombosis), 30-day and 90-day mortality, Readmission rate, Reoperation rate, and cost/efficacy analysis.

\section{Participant timeline $\{13\}$}

Participant timeline is presented in SPIRIT Table 1. Participant has priority when admission and is well scheduled during follow-up.

\section{Sample size $\{14\}$}

The primary outcome of this RCT is the success rate of splenic vessel-preserving (Kimura) approach. A $42.1 \%$ of splenic vessel-preserving rate via laparoscopic approach has been reported previously from the investigators' institution ${ }^{8}$. Meanwhile, a $72.3 \%$ of splenic vessel-preserving rate via robotic approach has been retrospectively reported by Chen et al. from Ruijin hospital ${ }^{11}$, which has similar surgical volume and experience in pancreatectomy with the investigators' hospital. We calculated that 67 patients each group (134 for total, including $5 \%$ of expulsion rate) are needed to detect an expected $25 \%$ increase in splenic vessel-preserving rate, with a power of $80 \%(1-\beta)$ and a 2 -sided a level of 0.05 . The $25 \%$ increase was based on our own data and reported literature. The formula is listed in the following (see Supplemental content 3):

$$
\mathrm{N}=\frac{2 \times 0.5(P 1+P 2) \times 0.5[(1-P 1)+(1-P 2)] \times\left(Z_{\alpha}+Z_{\beta}\right)^{2}}{(P 1-P 2)^{2}}
$$

" $\mathrm{N}$ " represents sample size for each group. A parallel design is adapted in this study, and ratio of the two groups is $1: 1$. Type I error is set as $5 \%, a=0.05, Z_{\alpha}=1.96 . \beta=0.2, Z_{\beta}=0.84$. Power of test is $1-\beta=80 \%$. $P 1$ and $\mathrm{P} 2$ represent the estimated splenic vessel-preserving rate of the observational group and the control group, respectively. $\mathrm{P} 1=67 \%, \mathrm{P} 2=42 \%$.

\section{Recruitment \{15\}}

All candidate patients for planned SPDP from surgical clinic will be recruited in the trial. Based on the huge amount of outpatients, the investigators have confidence to recruit enough subjects as planned. 
Also, the investigators plan to launch recruitment campaign to attract candidates and referrals from other hospitals or specialties.

\section{Assignment of interventions: allocation}

\section{Sequence generation $\{16 a\}$}

A series of consecutive random number is generated by SPSS software version 22.0. Randomization will be performed between RSPDP and LSPDP with equal assignment to the two groups (1:1). An independent co-investigator takes charge in the randomization progress (Fig. 1).

\section{Concealment mechanism $\{16 \mathrm{~b}\}$}

A random allocation sequence is generated by SPSS software. Allocation numbers and related information are concealed in sequentially numbered, opaque, sealed envelope. Interventions are assigned to the enrolled participants according to the sequence.

\section{Implementation $\{16 c\}$}

The central registry of the surgical department of $\mathrm{PUMCH}$ takes charge in the allocation sequence generation, participants enrollment and assigning.

\section{Assignment of interventions: Blinding}

\section{Who will be blinded $\{17 \mathrm{a}\}$}

This is a patient-blinded trial, therefore the trial participants are blinded (participants are informed of minimally invasive procedures, but not informed of robotic or laparoscopic in detail). Also, the data analysts are blinded.

\section{Procedure for unblinding if needed $\{17 \mathrm{~b}\}$}

Not applicable, because this is a surgical approach-related trial, not about medicine.

\section{Data collection and management}

\section{Plans for assessment and collection of outcomes $\{18 \mathrm{a}\}$}

Data will be recorded in the CRF and finished before the participant is discharged. Subsequently, the data will be uploaded to the ResMan database, which we used to manage trial data.

\section{Plans to promote participant retention and complete follow-up $\{18 \mathrm{~b}\}$}

We will offer appointment register of clinic follow-up after the participants are discharged. If the participant cannot come for clinic, a telephone follow-up will be arranged. 


\section{Data management $\{19\}$}

Data entry will be double checked for data validity. The ResMan software is applied to guarantee the process of data coding, security, and storage. Once the recruitment is finished, all the data is analysed using SPSS ${ }^{\circledR}$ version 23.0 (IBM Corporation, Armonk, NY, USA).

\section{Confidentiality $\{27\}$}

Only researchers, institution employee, and ethics committees get the access to the participants' records. Participants' identities will be concealed in any research related publishes.

Plans for collection, laboratory evaluation and storage of biological specimens for genetic or molecular analysis in this trial/future use $\{33\}$

There is no plan for collecting additional biological specimens in this trial.

\section{Statistical methods}

\section{Statistical methods for primary and secondary outcomes $\{20 \mathrm{a}\}$}

All statistical analyses will be performed with SPSS software. Continuous variables will be expressed as median (range) or mean(SD), and categorical variables will be shown as frequencies and percentages. Categorical variables (such as the primary outcome in this study) are going to be analysed by means of $\chi 2$ or Fisher's exact test, and Student's $t$ test or Non-parameter test is going to be used for continuous variables. $P<0.05$ is considered statistically significant.

\section{Interim analyses $\{21 \mathrm{~b}\}$}

Interim analysis will be performed when 80 participants are enrolled. If the success rate of Kimura procedure is obviously increased in the robotic group compared with that of the laparoscopic group, termination of the trial will be discussed by the PI and teammates and reported to the ethics board.

\section{Methods for additional analyses (e.g. subgroup analyses) \{20b\}}

Not applicable.

Methods in analysis to handle protocol non-adherence and any statistical methods to handle missing data $\{20 \mathrm{c}\}$

Enrolled participants will be excluded if they do not accept randomization or receive intervention after randomization. Only participant missing key data (eg, do not record the intraoperative information) will be excluded.

Plans to give access to the full protocol, participant level-data and statistical code $\{31 \mathrm{c}\}$ 
We plan to share the data to the public within 6 months after finished the trial.

\section{Oversight and monitoring}

\section{Composition of the coordinating centre and trial steering committee $\{5 \mathrm{~d}\}$}

The data monitoring committee (DMC) work independently, which is consisted of principal investigator, data manager, data monitors, and statistical analysts.

\section{Composition of the data monitoring committee, its role and reporting structure $\{21 \mathrm{a}\}$}

The DMC supervise the process of the trial and check data recording, data validity during the trail. Also, the DMC monitor the interim analyses, if there is significant difference of the primary outcome between the two groups, it will report and discuss whether to cease the trial.

\section{Adverse event reporting and harms $\{22\}$}

In this trial, the intervention refers to different surgical approaches, not medicine. We will report any approach or instruments related complication (adverse event), including timing, reason, severity, treatment, and outcome of the adverse event.

\section{Frequency and plans for auditing trial conduct $\{23\}$}

Annually after recruiting the first participant.

Plans for communicating important protocol amendments to relevant parties (e.g. trial participants, ethical committees) $\{25\}$

If important change occurs in study protocol, a breach report form will be created and reported to the sponsor, institutional ethics committee, trial participants. Also, trial registry will be updated.

\section{Dissemination plans $\{31$ a $\}$}

We plan to publish high-quality research article in specialty journal after completing the trial.

\section{Discussion}

Robotic-assisted splenic preserving distal pancreatectomy have been reported increasingly. Compared with conventional laparoscopy, the robotic surgical system provides technical advantages such as magnified three-dimensional view, Endo-Wrist with seven degrees of freedom, and tremor elimination. However, it remains controversial that whether the technical advantages could be transferred into patients' benefit, especially splenic preserving rate.

Retrospective study of the authors' institution and published literature have revealed that RSPDP led to increased or at least similar splenic preservation rate, as well as short-term surgical complication rate. 
The possible reason for the increase lies in a better control of intraoperative bleeding for the robotic approach. Nonetheless, high quality evidence lacks to testify the potential advantage of the robotic approach. Herein, we launch this RCT to evaluate the efficacy and surgical outcomes of RSPDP.

In the present study, the success rate of Kimura approach was set as the primary outcome. Based on previous data, we assume a $25 \%$ increase in Kimura success rate of the RSPDP group over that of the LSPDP group. A total of 134 participants are needed to detect the difference. If these values are realized, the robotic approach could be the preferred choice for people in need.

\section{Trial status}

Institutional Ethics Committee of the Peking Union Medical College Hospital has approved the final version of the protocol (Version 2.0 on April 28, 2020, approval number: ZS-2329). It was registered on the China Clinical Trials Registry (ChiCTR2000029177). We recruited the first participant on June $1^{\text {st }}$, and the recruitment planed to be be completed in June 2022.

\section{Abbreviations}

DP: Distal pancreatectomy; SPDP:Spleen preserving distal pancreatectomy; RCT:Randomized controlled trial; RSPDP:Robotic assisted spleen preserving distal pancreatectomy; LSPDP:Laparoscopic spleen preserving distal pancreatectomy; OPSI:Overwhelming post-splenectomy infection; MCN:Mucinous cystic neoplasm; pNET:Pancreatic Neuroendocrine Tumor; IPMN:Intraductal papillary mucinous neoplasm; SPN:Solid pseudopapillary neoplasm; PUMCH:Peking Union Medical College Hospital; CONSORT:Consolidated Standards of Reporting Trials; CT:Computed tomography; SMV:Superior mesentery vein; PV:Portal vein; LOS:Length of hospital stay; DGE:Delayed gastric emptying; SPIRIT:Standard Protocol Items:Recommendations for Interventional Trials.

\section{Declarations}

\section{Acknowledgements}

Not Applicable.

\section{Authors' contributions \{31b\}}

TF and GJC designed the trial. TF calculated the sample size and wrote the manuscript, and GJC revised it. LCX and LJ reviewed the literature and assisted in CRF design. All authors have read and approved the manuscript.

\section{Funding $\{4\}$}

Not funded yet (In the process of application). 
Availability of data and materials $\{29\}$

Data will be open to the public within six months after completing the present trial.

\section{Ethics approval and consent to participate $\{24\}$}

Institutional Ethics Committee of the Peking Union Medical College Hospital has approved the final version of the protocol (Version 2.0 on April 28, 2020, approval number: ZS-2329). Informed consent will be obtained from all study participants.

\section{Consent for publication $\{32\}$}

Not Applicable.

\section{Competing interests $\{28\}$}

The authors declare no competing interests.

\section{References}

1. Hu Y-H, Qin Y-F, Yu D-D, et al. Meta-analysis of short-term outcomes comparing robot-assisted and laparoscopic distal pancreatectomy. Journal of comparative effectiveness research 2020; 9(3):201218.

2. Najafı N, Mintziras I, Wiese D, et al. A retrospective comparison of robotic versus laparoscopic distal resection and enucleation for potentially benign pancreatic neoplasms. Surgery today 2020:10.1007/s00595-020-01966-z.

3. Xu S-B, Jia C-K, Wang J-R, et al. Do patients benefit more from robot assisted approach than conventional laparoscopic distal pancreatectomy? A meta-analysis of perioperative and economic outcomes. Journal of the Formosan Medical Association = Taiwan yi zhi 2019; 118(1 Pt 2):268-278.

4. Kang $\mathrm{CM}$, Chung $\mathrm{YE}$, Jung MJ, et al. Splenic vein thrombosis and pancreatic fistula after minimally invasive distal pancreatectomy. Br J Surg 2014; 101(2):114-9.

5. Boselli C, Barberini F, Listorti C, et al. Distal pancreatectomy with splenic preservation: A short-term outcome analysis of the Warshaw technique. International journal of surgery (London, England) 2015; 21 Suppl 1:S40-S43.

6. Juo Y-Y, King JC. Robotic-assisted spleen preserving distal pancreatectomy: a technical review. Journal of visualized surgery 2017; 3:139-139.

7. Lee LS, Hwang HK, Kang CM, et al. Minimally Invasive Approach for Spleen-Preserving Distal Pancreatectomy: a Comparative Analysis of Postoperative Complication Between Splenic Vessel Conserving and Warshaw's Technique. Journal of gastrointestinal surgery : official journal of the Society for Surgery of the Alimentary Tract 2016; 20(8):1464-1470. 
8. Dai MH, Shi N, Xing C, et al. Splenic preservation in laparoscopic distal pancreatectomy. British Journal of Surgery 2017; 104(4):452-462.

9. Eckhardt S, Schicker C, Maurer E, et al. Robotic-Assisted Approach Improves Vessel Preservation in Spleen-Preserving Distal Pancreatectomy. Digestive surgery 2016; 33(5):406-413.

10. Hong S, Song KB, Madkhali AA, et al. Robotic versus laparoscopic distal pancreatectomy for leftsided pancreatic tumors: a single surgeon's experience of 228 consecutive cases. Surgical endoscopy 2019:10.1007/s00464-019-07047-8.

11. Chen S, Zhan Q, Chen J-Z, et al. Robotic approach improves spleen-preserving rate and shortens postoperative hospital stay of laparoscopic distal pancreatectomy: a matched cohort study. Surgical endoscopy 2015; 29(12):3507-3518.

12. Goh BKP, Chan CY, Soh H-L, et al. A comparison between robotic-assisted laparoscopic distal pancreatectomy versus laparoscopic distal pancreatectomy. The international journal of medical robotics + computer assisted surgery : MRCAS 2017; 13(1):10.1002/rcs.1733.

13. Suman P, Rutledge J, Yiengpruksawan A. Robotic distal pancreatectomy. JSLS : Journal of the Society of Laparoendoscopic Surgeons 2013; 17(4):627-635.

\section{Tables}

Table 1: Standard Protocol Items: Recommendations for Interventional Trials (SPIRIT) 


\begin{tabular}{|c|c|c|c|c|c|c|}
\hline & \multicolumn{6}{|c|}{ STUDY PERIOD } \\
\hline & Enrollment & Allocation & Surgery & Discharge & Post-allocation & Close-out \\
\hline Time point & -30 days & 0 & $\begin{array}{l}1-3 \\
\text { days }\end{array}$ & $0-21$ days & 60 days & 90 days \\
\hline \multicolumn{7}{|l|}{ ENROLLMEN: } \\
\hline Eligibility screen & $x$ & & & & & \\
\hline Informed consent & $x$ & & & & & \\
\hline Allocation & & $x$ & & & & \\
\hline \multicolumn{7}{|l|}{ INTERVENTIONS: } \\
\hline RSPDP & & 4 & $\longrightarrow$ & & & \\
\hline LSPDP & & & $\rightarrow$ & & & \\
\hline \multicolumn{7}{|l|}{ ASSESSMENTS: } \\
\hline$H \& P$ & $x$ & & & $x$ & $x$ & $x$ \\
\hline Blood test & $x$ & & & $x$ & $x$ & $x$ \\
\hline Ultrasonography & $x$ & & & $x$ & $x$ & $x$ \\
\hline (Enhanced) $\quad \mathrm{CT}$ & $x$ & & & $x$ & & $x$ \\
\hline scan & & & $x$ & & & \\
\hline Surgical record & & & & $x$ & & \\
\hline Postoperative & & & & $x$ & & \\
\hline $\begin{array}{l}\text { record } \\
\text { Pathological } \\
\text { findings }\end{array}$ & & & & & & \\
\hline
\end{tabular}

Figures 


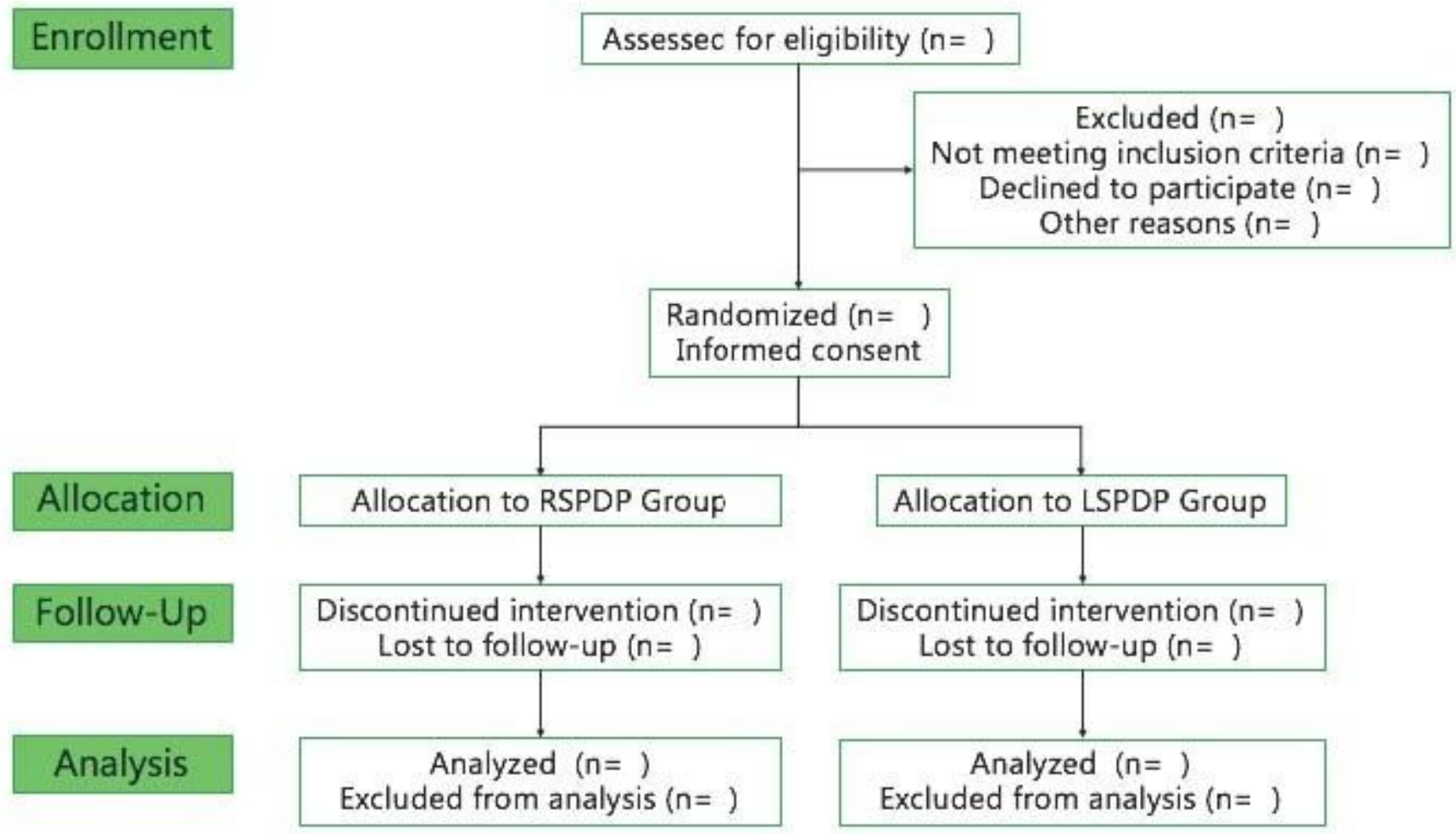

\section{Figure 1}

Besides enrollment, patients' allocation, follow-up and data analysis are also performed in PUMCH 